\title{
DYNAMICS OF PHYSICAL TRAINING OF TABLE TENNIS PEOPLE IN PRIMARY PREPARATORY GROUPS
}

\author{
Dilrabo Abdisamatovna Umurkulova
}

Master's Degree Student, Uzbek State University Of Physical Culture And Sports, Uzbekistan

\section{ABSTRACT}

This article presents the results of pedagogical experiments on the effectiveness of special exercises in improving the physical fitness of table tennis players aged 10-12 (boys and girls) engaged in the initial stage of preparation.

KEYWORDS:- Initial training phase, physical training, physical development, physical load, endurance, test criteria.

\section{INTRODUCTION}

Today, the popularization of physical culture and sports has been identified by the world community as one of the important directions of social policy. The achievements in this area will introduce the country to the world and give pride to all our compatriots. It should be noted that in the development of children's and adolescents' sports in the world, the involvement of gifted children in sports, training their sports skills, assessing their psychological state, determining the level of development of morphofunctional indicators, improving the physical, technical and tactical training. - A lot of research work has been done on the organization of training on a scientific basis.

After the independence of our country, the necessary conditions have been created for regular physical culture and sports. Modern sports complexes have been built in cities and villages. In order to ensure the implementation of the Resolution of the President of the Republic of Uzbekistan Sh.M.Mirziyoev dated June 3, 2017 No PP-3031 "On measures for further development of physical culture and mass sports" the focus is a confirmation of the above considerations. Thus, the development of mass sports, especially children's sports on a scientific basis, indicates the need to pay special attention to the creation of its organizational and technological basis.

Based on this, it can be said that the chosen topic of the research work is one of the topical issues.

Literature analysis and methodology: The aim of the study was to determine the effectiveness of 
CURRENT RESEARCH JOURNAL OF PEDAGOGICS 2(11): 168-177,

November 2021 DOI: https://doi.org/10.37547/pedagogics-crjp-02-11-32

ISSN 2767-3278

(C)2021 Master Journals

Crossref do) 81 Google

Accepted 25th November, 2021 \& Published 30 ${ }^{\text {th }}$ November, 2021

special exercises to increase the physical fitness of table tennis players aged 10-12 years.

The development of physical qualities such as agility, coordination, agility, agility, flexibility and jumping in children aged 10-12 years is one of the main tasks of each trainer-teacher $[2,5]$. These physical qualities allow trainees to feel free in any life situation, to determine their social and living conditions and their worthy place in sports early. The development of these qualities, in turn, determines the level of physical fitness of practitioners of this age.

In sports practice, physical fitness is assessed using a number of exercise and test criteria. In our study in the BOSM primary training group, running 30 meters from a high start; $3 \times 10$ meter sprint; throwing a stuffing ball weighing 1 $\mathrm{kg}$; leaning forward while sitting; 6-minute run; long jump from standing position; we used control tests such as pulling on a high bar (girls on a low bar) and catching a falling line. The test results allowed to obtain the necessary information about the level of development of physical fitness of boys and girls in the primary training group.
Results: At the beginning of the pedagogical experiment, no statistical differences in physical fitness of 10-year-old boys were observed between the experimental and control groups. At the end of the pedagogical experiment, 11-12year-old boys ran 30 meters from the high start, $3 \times 10$ meters jogging, pulling, throwing a stuffed ball weighing $1 \mathrm{~kg}$, long jump from a standing position, sitting forward, bending forward, catching the descending line, 6-minute run a level of reliability $(\mathrm{P}<0.05)$ was observed between the experimental and control groups on the tests (Table 1).

At the beginning of the pedagogical experiment, no statistical differences were observed in all tests on physical fitness in 10-year-old girls between the experimental and control groups. At the end of the pedagogical experiment, 11-12 year old girls ran 30 meters from the high start, 3 $\times 10$ meters jogging, pulling on a low barbell, throwing a stuffing ball weighing $1 \mathrm{~kg}$, long jump from a standing position, bending forward while sitting, catching a falling line, 6 statistically differentiated on minute running tests (Table 2).

Table 1

Experimental and control groups Indicators of physical fitness at the beginning and end of training in boys aged 10-12 years

$[\mathrm{n}=38]$

\begin{tabular}{|c|c|c|c|c|c|}
\hline \multirow{2}{*}{ TNo } & & \multicolumn{2}{|c|}{ Groups } & Degree of \\
confidenc \\
\cline { 3 - 5 }
\end{tabular}


CURRENT RESEARCH JOURNAL OF PEDAGOGICS 2(11): 168-177,

November 2021 DOI: https://doi.org/10.37547/pedagogics-crjp-02-11-32

ISSN 2767-3278

(C)2021 Master Journals

Crossref dol 81 Google

Accepted 25th November, 2021 \& Published 30th November, 2021

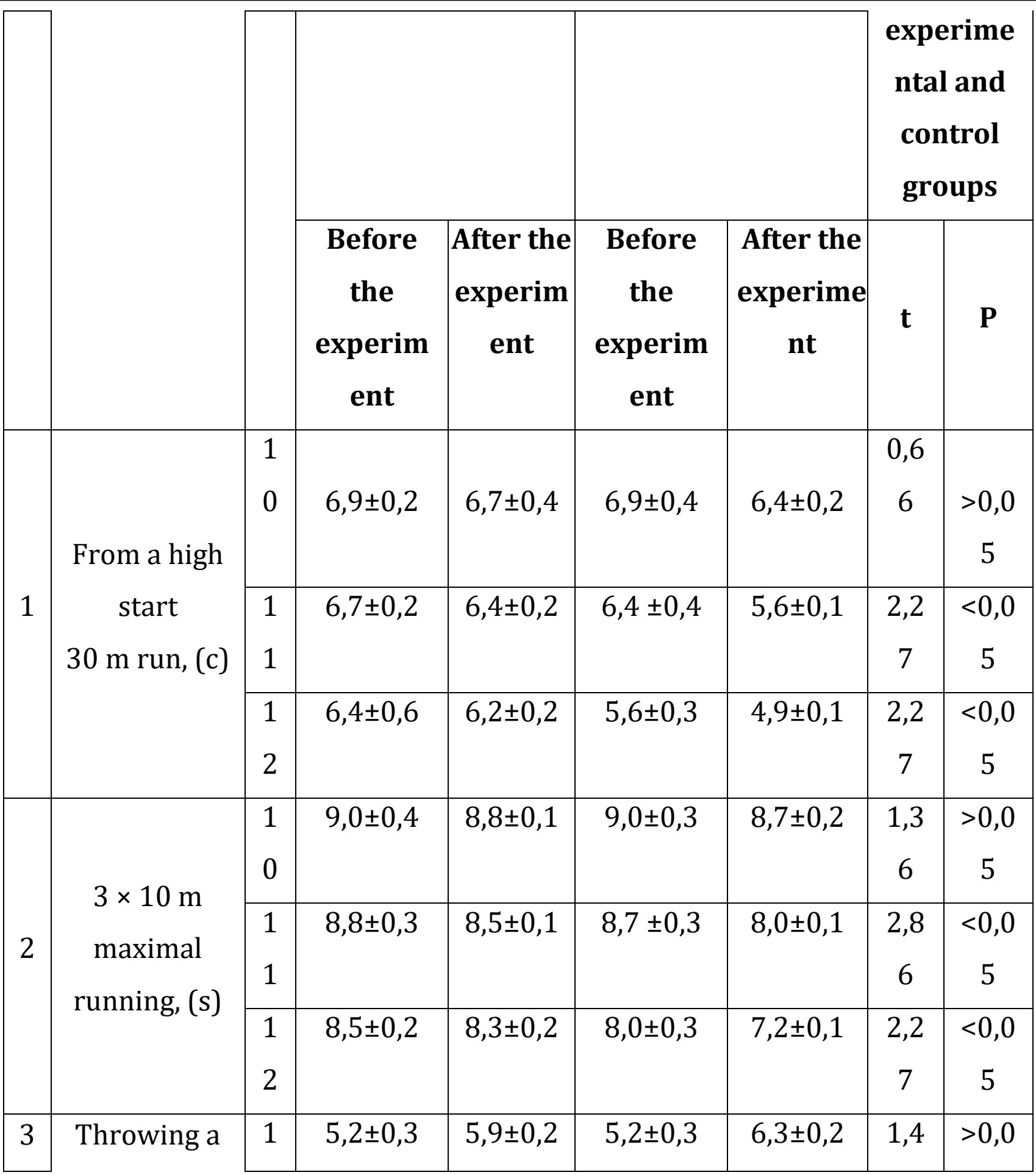


CURRENT RESEARCH JOURNAL OF PEDAGOGICS 2(11): 168-177,

November 2021 DOI: https://doi.org/10.37547/pedagogics-crjp-02-11-32

ISSN 2767-3278

(C)2021 Master Journals

Crossref do: 81 Google

Accepted 25th November, 2021 \& Published 30 ${ }^{\text {th }}$ November, 2021

\begin{tabular}{|c|c|c|c|c|c|c|c|c|}
\hline & stuffed ball & 0 & & & & & 2 & 5 \\
\hline & (1 kg) & 1 & $5,9 \pm 0,4$ & $6,8 \pm 0,3$ & $6,3 \pm 0,7$ & $7,9 \pm 0,1$ & 2,1 & $<0,0$ \\
\hline & & 1 & & & & & 8 & 5 \\
\hline & & 1 & $6,8 \pm 0,8$ & $7,8 \pm 0,2$ & $7,9 \pm 0,7$ & $9,5 \pm 0,2$ & 2,1 & $<0,0$ \\
\hline & & 2 & & & & & 4 & 5 \\
\hline & Long & 1 & $157,2 \pm 2,2$ & $168,4 \pm$ & 157,3 & $169,3 \pm 2$ & 0,1 & $>0,0$ \\
\hline & jumping & 0 & & 7,6 & $\pm 5,0$ & 0 & 0 & 5 \\
\hline & from a & 1 & $168,4 \pm 7,8$ & $180,2 \pm$ & $169,3 \pm 6,1$ & $182,5 \pm 0$ & 2,2 & $<0,0$ \\
\hline & standing & 1 & & 0,6 & & 2 & 2 & 5 \\
\hline & position & 1 & $180,2 \pm 9,4$ & $192,1 \pm$ & $182,5 \pm 6,0$ & $196,9 \pm 0$ & 2,3 & $<0,0$ \\
\hline & $(\mathrm{cm})$. & 2 & & 0,8 & & 7 & 6 & 5 \\
\hline & & 1 & $9,7 \pm 0,2$ & $11,1 \pm 1$ & $9,7 \pm 0,9$ & $12,3 \pm 0,6$ & 1,0 & $>0,0$ \\
\hline & Leaning & 0 & & 0 & & & 8 & 5 \\
\hline - & forward & 1 & $11,1 \pm 1,1$ & $12,9 \pm 0$, & $12,3 \pm 0,8$ & $14,8 \pm 0,2$ & 2,5 & $<0,0$ \\
\hline & while sitting, & 1 & & 2 & & & 0 & 5 \\
\hline & $(\mathrm{cm})$ & 1 & $12,9 \pm 0,9$ & $14,9 \pm 0$ & $14,8 \pm 0,8$ & $17,7 \pm 0,3$ & 2,1 & $<0,0$ \\
\hline & & 2 & & 3 & & & 4 & 5 \\
\hline & & 1 & $3,5 \pm 0,6$ & $4,4 \pm 0,5$ & $3,5 \pm 0,8$ & $5,2 \pm 0,6$ & 1,0 & $>0,0$ \\
\hline & Pulling on a & 0 & & & & & 2 & 5 \\
\hline 6 & high barbell, & 1 & $4,4 \pm 0,8$ & $5,4 \pm 0,2$ & $5,2 \pm 0,7$ & $6,9 \pm 0,2$ & 2,5 & $<0,05$ \\
\hline & (times). & 1 & & & & & 0 & \\
\hline & & 1 & $5,4 \pm 0,9$ & $6,5 \pm 0,2$ & $6,9 \pm 0,8$ & $8,7 \pm 0,2$ & 2,5 & $<0,05$ \\
\hline
\end{tabular}


CURRENT RESEARCH JOURNAL OF PEDAGOGICS 2(11): 168-177,

November 2021 DOI: https://doi.org/10.37547/pedagogics-crjp-02-11-32

ISSN 2767-3278

(C)2021 Master Journals

Crossref dof Google

Accepted 25th November, 2021 \& Published 30th November, 2021

\begin{tabular}{|c|c|c|c|c|c|c|c|c|}
\hline & & 2 & & & & & 0 & \\
\hline \multirow{3}{*}{7} & \multirow{3}{*}{$\begin{array}{l}\text { Catch the } \\
\text { falling ruler, } \\
\text { (cm). }\end{array}$} & 0 & $20,9 \pm 2,2$ & $\begin{array}{c}19,8 \pm 0, \\
4\end{array}$ & $20,9 \pm 0,9$ & $18,8 \pm 0,8$ & $\begin{array}{c}1,0 \\
1\end{array}$ & $\begin{array}{c}>0,0 \\
5\end{array}$ \\
\hline & & 1 & $19,8 \pm 0,5$ & $\begin{array}{c}18,6 \pm 0 \\
3\end{array}$ & $18,8 \pm 0,9$ & $16,7 \pm 0,3$ & $\begin{array}{c}2,1 \\
4\end{array}$ & $\begin{array}{c}<0,0 \\
5\end{array}$ \\
\hline & & 2 & $18,6 \pm 0,5$ & $\begin{array}{c}17,3 \pm 0, \\
4\end{array}$ & $16,7 \pm 0,7$ & $14,0 \pm 0,5$ & $\begin{array}{c}2,1 \\
8\end{array}$ & $<0,05$ \\
\hline \multirow{3}{*}{8} & \multirow{3}{*}{$\begin{array}{l}\text { 6-minute } \\
\text { run, (m). }\end{array}$} & 0 & $\begin{array}{c}855,5 \pm 10 \\
3\end{array}$ & $\begin{array}{c}900,2 \pm \\
9,0\end{array}$ & $\begin{array}{c}855,6 \pm 12 \\
9\end{array}$ & $\begin{array}{c}910,2 \pm 8 \\
0\end{array}$ & $\begin{array}{c}0,8 \\
2\end{array}$ & $\begin{array}{c}>0,0 \\
5\end{array}$ \\
\hline & & 1 & $900,8 \pm 8,5$ & $\begin{array}{c}1117,2 \\
\pm 9,6\end{array}$ & $910,2 \pm 8,2$ & $\begin{array}{c}1192,7 \pm 8 \\
3\end{array}$ & $\begin{array}{c}4,8 \\
4\end{array}$ & $\begin{array}{c}<0,00 \\
1\end{array}$ \\
\hline & & 2 & $\begin{array}{c}1117,3 \pm 9 \\
2\end{array}$ & $\begin{array}{c}1340,5 \\
\pm 8,2\end{array}$ & $\begin{array}{c}1192,8 \pm 11 \\
, 1\end{array}$ & $\begin{array}{c}1484,3 \pm 7 \\
2\end{array}$ & $\begin{array}{c}6,2 \\
6\end{array}$ & $\begin{array}{c}<0,00 \\
1\end{array}$ \\
\hline
\end{tabular}

\section{Table 2}

Experimental and control groups Indicators of physical fitness at the beginning and end of training in girls aged 10-12 years $[n=36]$ 
CURRENT RESEARCH JOURNAL OF PEDAGOGICS 2(11): 168-177,

November 2021 DOI: https://doi.org/10.37547/pedagogics-crjp-02-11-32

ISSN 2767-3278

(C)2021 Master Journals

Crossref doi) 81 Google

Accepted 25th November, 2021 \& Published 30th November, 2021

\begin{tabular}{|c|c|c|c|c|c|c|c|c|}
\hline \multirow{3}{*}{ № } & \multirow{3}{*}{ Tests } & \multirow{3}{*}{ م્ } & \multicolumn{4}{|c|}{ Groups } & \multirow{2}{*}{\multicolumn{2}{|c|}{$\begin{array}{c}\text { Level of } \\
\text { reliability } \\
\text { between } \\
\text { experime } \\
\text { nt and } \\
\text { control } \\
\text { groups }\end{array}$}} \\
\hline & & & \multicolumn{2}{|c|}{ Control } & \multicolumn{2}{|c|}{ Experiment } & & \\
\hline & & & $\begin{array}{c}\text { Before } \\
\text { the } \\
\text { experim } \\
\text { ent }\end{array}$ & \begin{tabular}{|} 
After the \\
experime \\
nt
\end{tabular} & $\begin{array}{c}\text { Before the } \\
\text { experime } \\
\text { nt }\end{array}$ & $\begin{array}{c}\text { After the } \\
\text { experime } \\
\text { nt }\end{array}$ & $\mathbf{t}$ & $\mathbf{P}$ \\
\hline \multirow{3}{*}{1} & \multirow{3}{*}{$\begin{array}{c}\text { From a } \\
\text { high } \\
\text { start } \\
30 \mathrm{~m} \\
\text { run, (c) }\end{array}$} & $\begin{array}{l}1 \\
0\end{array}$ & $8,9 \pm 0,2$ & $8,7 \pm 0,1$ & $8,9 \pm 0,3$ & $8,3 \pm 0,2$ & $\begin{array}{c}1,8 \\
1\end{array}$ & $\begin{array}{c}>0,0 \\
5\end{array}$ \\
\hline & & $\begin{array}{l}1 \\
1\end{array}$ & $8,7 \pm 0,3$ & $8,4 \pm 0,1$ & $8,3 \pm 0,3$ & $7,6 \pm 0,1$ & $\begin{array}{c}2,8 \\
5\end{array}$ & $\begin{array}{c}<0,0 \\
5\end{array}$ \\
\hline & & $\begin{array}{l}1 \\
2\end{array}$ & $8,4 \pm 0,2$ & $8,2 \pm 0,1$ & $7,6 \pm 0,2$ & $6,9 \pm 0,2$ & $\begin{array}{c}2,2 \\
7\end{array}$ & $\begin{array}{c}<0,0 \\
5\end{array}$ \\
\hline \multirow{2}{*}{2} & \multirow{2}{*}{$\begin{array}{l}3 \times 10 \mathrm{~m} \\
\text { maximal } \\
\text { running, } \\
\text { (s) }\end{array}$} & $\begin{array}{l}1 \\
0\end{array}$ & $10,1 \pm 0,4$ & $9,9 \pm 0,2$ & $10,0 \pm 0,2$ & $9,6 \pm 0,2$ & $\begin{array}{c}0,7 \\
1\end{array}$ & $\begin{array}{c}>0,0 \\
5\end{array}$ \\
\hline & & $\begin{array}{l}1 \\
1\end{array}$ & $9,9 \pm 0,3$ & $9,7 \pm 0,1$ & $9,6 \pm 0,2$ & $9,0 \pm 0,1$ & $\begin{array}{c}2,8 \\
5\end{array}$ & $\begin{array}{c}<0,0 \\
5\end{array}$ \\
\hline
\end{tabular}


CURRENT RESEARCH JOURNAL OF PEDAGOGICS 2(11): 168-177,

November 2021 DOI: https://doi.org/10.37547/pedagogics-crjp-02-11-32

ISSN 2767-3278

(C)2021 Master Journals

Crossref dof 81 Google

Accepted 25 ${ }^{\text {th }}$ November, 2021 \& Published 30 ${ }^{\text {th }}$ November, 2021

\begin{tabular}{|c|c|c|c|c|c|c|c|c|}
\hline & & $\begin{array}{l}1 \\
2\end{array}$ & $9,7 \pm 0,4$ & $9,4 \pm 0,1$ & $9,0 \pm 0,3$ & $8,2 \pm 0,2$ & $\begin{array}{c}2,2 \\
7\end{array}$ & $\begin{array}{c}<0,0 \\
5\end{array}$ \\
\hline \multirow{3}{*}{3} & \multirow{3}{*}{$\begin{array}{l}\text { Throwin } \\
\text { g a } \\
\text { stuffed } \\
\text { ball (1 } \\
\text { kg) }\end{array}$} & $\begin{array}{l}1 \\
0\end{array}$ & $4,8 \pm 0,4$ & $5,5 \pm 0,2$ & $4,8 \pm 0,4$ & $5,8 \pm 0,2$ & $\begin{array}{c}1,0 \\
7\end{array}$ & $\begin{array}{c}>0,0 \\
5\end{array}$ \\
\hline & & $\begin{array}{l}1 \\
1\end{array}$ & $5,5 \pm 0,8$ & $6,3 \pm 0,1$ & $5,8 \pm 0,6$ & $7,3 \pm 0,3$ & $\begin{array}{c}2,1 \\
8\end{array}$ & $\begin{array}{c}<0,0 \\
5\end{array}$ \\
\hline & & 2 & $6,3 \pm 0,8$ & $7,2 \pm 0,2$ & $7,3 \pm 0,6$ & $8,8 \pm 0,2$ & $\begin{array}{c}2,1 \\
4\end{array}$ & $\begin{array}{c}<0,0 \\
5\end{array}$ \\
\hline \multirow{3}{*}{4} & \multirow{3}{*}{$\begin{array}{c}\text { Long } \\
\text { jumping } \\
\text { from a } \\
\text { standing } \\
\text { position } \\
\text { (cm). }\end{array}$} & $\begin{array}{l}1 \\
0\end{array}$ & $152,0 \pm 5,0$ & $\begin{array}{c}162,6 \pm 1 \\
6\end{array}$ & $152,0 \pm 6,0$ & $\begin{array}{c}163,2 \pm 2 \\
0\end{array}$ & $\begin{array}{c}0,2 \\
3\end{array}$ & $\begin{array}{c}>0,0 \\
5\end{array}$ \\
\hline & & $\begin{array}{l}1 \\
1\end{array}$ & $162,6 \pm 5,8$ & $\begin{array}{c}173,5 \pm 0 \\
6\end{array}$ & $163,4 \pm 4,5$ & $\begin{array}{c}175,9 \pm 0 \\
3\end{array}$ & $\begin{array}{c}2,3 \\
8\end{array}$ & $\begin{array}{c}<0,0 \\
5\end{array}$ \\
\hline & & 1 & $173,5 \pm 5,2$ & $\begin{array}{c}184,6 \pm 0 \\
6\end{array}$ & $175,9 \pm 4,6$ & $\begin{array}{c}189,4 \pm 0 \\
9\end{array}$ & $\begin{array}{c}2,2 \\
2\end{array}$ & $\begin{array}{c}<0,0 \\
5\end{array}$ \\
\hline \multirow{2}{*}{5} & \multirow{2}{*}{$\begin{array}{l}\text { Leaning } \\
\text { forward } \\
\text { while } \\
\text { sitting, }\end{array}$} & 0 & $9,9 \pm 1,1$ & $11,1 \pm 1,0$ & $9,9 \pm 1,0$ & $12,0 \pm 0,5$ & $\begin{array}{c}0,8 \\
0\end{array}$ & $\begin{array}{c}>0,0 \\
5\end{array}$ \\
\hline & & 1 & $11,1 \pm 1,2$ & $13,0 \pm 0,3$ & $12,0 \pm 1,1$ & $15,0 \pm 0,4$ & $\begin{array}{c}2,2 \\
0\end{array}$ & $<0,0$ \\
\hline
\end{tabular}


CURRENT RESEARCH JOURNAL OF PEDAGOGICS 2(11): 168-177,

November 2021 DOI: https://doi.org/10.37547/pedagogics-crjp-02-11-32

ISSN 2767-3278

(C)2021 Master Journals

Crossref dof 81 Google

Accepted 25 ${ }^{\text {th }}$ November, 2021 \& Published 30 ${ }^{\text {th }}$ November, 2021

\begin{tabular}{|c|c|c|c|c|c|c|c|c|}
\hline & $(\mathrm{cm})$. & & & & & & & 5 \\
\hline & & $\begin{array}{l}1 \\
2\end{array}$ & $13,0 \pm 1,0$ & $15,1 \pm 0,3$ & $15,0 \pm 0,9$ & $18,8 \pm 0,7$ & $\begin{array}{c}2,2 \\
3\end{array}$ & $\begin{array}{c}<0,0 \\
5\end{array}$ \\
\hline & & $\begin{array}{l}1 \\
0\end{array}$ & $2,2 \pm 0,8$ & $3,0 \pm 0,4$ & $2,2 \pm 0,9$ & $3,3 \pm 0,5$ & $\begin{array}{c}0,4 \\
6\end{array}$ & $\begin{array}{c}>0,0 \\
5\end{array}$ \\
\hline 6 & $\begin{array}{c}\text { pulling } \\
\text { on a high } \\
\text { barbell, }\end{array}$ & $\begin{array}{l}1 \\
1\end{array}$ & $3,0 \pm 0,6$ & $3,8 \pm 0,4$ & $3,3 \pm 0,8$ & $5,5 \pm 0,5$ & $\begin{array}{c}2,1 \\
9\end{array}$ & $\begin{array}{c}<0,0 \\
5\end{array}$ \\
\hline & (times) & $\begin{array}{l}1 \\
2\end{array}$ & $3,8 \pm 0,8$ & $4,8 \pm 0,4$ & $5,5 \pm 0,7$ & $7,6 \pm 0,3$ & $\begin{array}{c}2,2 \\
0\end{array}$ & $\begin{array}{c}<0,0 \\
5\end{array}$ \\
\hline & & $\begin{array}{l}1 \\
0\end{array}$ & $24,0 \pm 0,8$ & $22,9 \pm 0,4$ & $24,0 \pm 0,4$ & $22,6 \pm 0,8$ & $\begin{array}{c}0,3 \\
4\end{array}$ & $\begin{array}{c}>0,0 \\
5\end{array}$ \\
\hline 7 & falling & 1 & $22,9 \pm 0,6$ & $21,6 \pm 0,4$ & $22,6 \pm 0,7$ & $20,2 \pm 0,3$ & 2,2 & $<0,0$ \\
\hline & (cing). & 2 & $21,6 \pm 0,5$ & $20,2 \pm 0,5$ & $20,2 \pm 0,9$ & $17,6 \pm 0,2$ & $\begin{array}{c}2,2 \\
2\end{array}$ & $\begin{array}{c}<0,0 \\
5\end{array}$ \\
\hline 8 & $\begin{array}{l}\text { 6-minute } \\
\text { run, (m). }\end{array}$ & 1 & $\begin{array}{c}810,5 \pm 10 \\
3\end{array}$ & $\begin{array}{c}852,2 \pm 9 \\
6\end{array}$ & $\begin{array}{c}810,6 \pm 18 \\
9\end{array}$ & $\begin{array}{c}870,2 \pm 8 \\
0\end{array}$ & $\begin{array}{c}1,4 \\
3\end{array}$ & $\begin{array}{c}>0,0 \\
5\end{array}$ \\
\hline
\end{tabular}


CURRENT RESEARCH JOURNAL OF PEDAGOGICS 2(11): 168-177,

November 2021 DOI: https://doi.org/10.37547/pedagogics-crjp-02-11-32

ISSN 2767-3278

(C)2021 Master Journals

Crossref dof 81 Google

Accepted 25th November, 2021 \& Published 30 ${ }^{\text {th }}$ November, 2021

\begin{tabular}{|l|c|c|c|c|c|c|c|}
\hline & 1 & $852,8 \pm 12$, & $30,7 \pm 10$, & $870,2 \pm 17$, & $998,7 \pm 19$, & 2,3 & \\
& 1 & 1 & 7 & 2 & 2 & 0 & $<0,0$ \\
& & & & & & & 5 \\
\hline & 1 & $930,7 \pm 12$, & $1012,2 \pm 4$ & $999,5 \pm 20$, & $1174,8 \pm 16$ & 2,1 & $<0,0$ \\
& 2 & 2 & 0,8 & 8 &, 2 & 4 & 5 \\
\hline
\end{tabular}

\section{DisCUSSION}

A study of the physical fitness of 11-year-old boys in the experimental and control groups revealed significant changes in boys in the experimental group compared to those in the control group. In particular, statistical differences between the experimental and control groups included 6-minute running, $3 \times 10$ meter sprint $(\mathrm{P}<0.001)$, throwing a $1 \mathrm{~kg}$ ball, long jump from a standing position, bending forward while sitting, pulling on a high barbell, descending line. a statistical difference $(\mathrm{P}<0.05)$ was found in the control tests for catch and run 30 meters from the high start.

When the physical fitness of 12-year-old boys in the experimental and control groups was studied, there were significant changes in the children in the experimental group compared to those in the control group. In particular, statistical differences between the experimental and control groups included 6-minute running ( $\mathrm{P}$ $<0.001$ ), 30-meter run from the high start, $3 \times$ 10 -meter sprint, throwing a $1 \mathrm{~kg}$ stuffed ball, long jump from a standing position, sitting forward, there was a statistical difference $(\mathrm{P}<0.05)$ in the control tests of traction and catching the descending line on the high bar.

Experimental and control groups No static differences were observed in control tests of the level of development of physical qualities in 10- year-old girls. At the end of the experiment, no statistical differences were observed $(\mathrm{P}>0.05)$, excluding the 6 -minute run $(\mathrm{P}<0.01)$ between the experiment and the control group.

When the physical fitness of 11-year-old girls in the experimental and control groups was studied, there were significant changes in the experimental group girls compared to the boys in the control group. Basically, statistical differences between the experimental and control groups were observed in a $3 \times 10$ meter sprint, throwing a $1 \mathrm{~kg}$ padding ball, long jump from a standing position, bending forward while sitting, pulling on a high barbell, catching a descending line, and running 30 meters from a high start.

\section{Conclusion}

During the experiment, the physical fitness of 10-12-year-old boys and girls, the confidence level between 10-11-year-old boys in the experimental and control groups on the $30 \mathrm{~m}$ running test from the high start, $\mathrm{t}=2.27$, $(\mathrm{R}$ $<0.05)$, in girls while $\mathrm{t}=2.85,(\mathrm{R}<0.05)$, respectively, among boys aged 11-12 years $t=$ 2.27, $(\mathrm{R}<0.05)$, and among girls $\mathrm{t}=2.27$, $(\mathrm{R}<$ $0.05)$. It was also proved experimentally that the level of reliability $(\mathrm{R}<0.05)$ increased differently among boys and girls aged 10-11, 11-12 years on all 8 tests.

According to the results obtained, the level of 
CURRENT RESEARCH JOURNAL OF PEDAGOGICS 2(11): 168-177,

November 2021 DOI: https://doi.org/10.37547/pedagogics-crjp-02-11-32

ISSN 2767-3278

(C)2021 Master Journals

Crossref dof 81 Google

Accepted 25th November, 2021 \& Published 30th November, 2021

physical fitness of 10-12-year-old children in the primary training group was not statistically significant $(R>0.05)$ for all 7 tests except for the 6-minute run $(\mathrm{R}<0.001)$.

\section{REFERENCES}

1. Моргунова И.И. Настольный теннис и методика обучения. - Т.: Пойтахт-Принт, 2013. - 128 c.

2. Xalmatova N. Methods of teaching table tennis to students. -- Tashkent. UMIDDEZIGN, 2020. - 55 p.

3. Железняка Ю.Д, Портнова Ю.М. Спортивные игры: техника, тактика, методика обучения. - М.: Академия, 2004. 520 c.

4. Ne'matov B.I. Dynamics of assessment of the level of physical fitness of participants in the primary training groups of children and youth sports school. // Fan-sportga. Toshkent, 2018. -№1.- C. Pp. 22-26. 